\title{
Interpolatory Inequalities for First Kind Convolution Volterra Integral Equations
}

\author{
$\underline{\text { M. Hegland }}^{\mathrm{a}}$ and R.S. Anderssen ${ }^{\mathrm{b}}$ \\ ${ }^{a}$ Mathematical Sciences Institute, The Australian National University, Acton, ACT, 2601 \\ ${ }^{\mathrm{b}}$ CSIRO, Australia \\ Email: Markus.Hegland@anu.edu.au
}

Abstract: We consider the problem of computing a function $u(t)$ which satisfies the equation

$$
k * u=\int_{0}^{t} k(t-s) u(s) d s=f(t), \quad 0 \leq t<\infty,
$$

where $k(t)$ is a given kernel and the right-hand side $f(t)$ is only known through some observations which contain observational errors. This problem arises in the study of the rheology of linear viscoelastic materials. It is well known that solving this first kind Volterra integral equation is ill-posed and thus special regularisation techniques are required to solve it in a stable fashion.

An important property of many kernels occurring in rheological applications is that they admit solutions $h(t)$ of the interconversion equation

$$
\int_{0}^{t} k(t-s) h(s) d s=t, \quad 0 \leq t<\infty .
$$

In such situations, the solution of the Volterra equation takes the following form

$$
u(t)=\frac{d^{2}}{d t^{2}}\left\{\int_{0}^{t} h(t-s) f(s) d s\right\} .
$$

Problems where the interconversion equation can be solved explicitly includes

$$
k(t)=1+\sum_{j=1}^{n} a_{j} \exp \left(-t / \tau_{j}\right)
$$

with the $\tau_{j}>0$. A characterisation of the solutions $h(t)$ for such $k(t)$ will be given below.

Even when the solution $h(t)$ to the interconversion equation is known, the problem of computing the solution of (a) as (c) is still ill-posed. A consequence is that regularisation techniques are required to compute $u(t)$. Even then for data containing errors, one can at best get error bounds of the form

$$
\left\|u_{\epsilon}-u\right\| \leq \eta(\epsilon) \epsilon
$$

for some unbounded $\eta(\epsilon)$. The form of such error bounds based, on interpolatory inequalities, will be discussed for the first kind Volterra equations considered here.

Finally, a numerical technique to solve such Volterra equations, when the data is sampled and contains errors, will be discussed. The method is obtained from equation (c) by explicit differentiation, it evaluates

$$
u(t)=h^{\prime}(0) f(t)+\int_{0}^{t} h^{\prime \prime}(t-s) f(s) d s+h(0) \frac{d f(t)}{d t},
$$

where the prime in $\left(h^{\prime}\right)$ denotes the derivative of the function $h$ etc. Instead of having to perform the numerical differentiation of the kernel $k$ in equation (a) and then solve the resulting second kind Volterra integral equation, or the numerical differentiation of equation (c), the solution can be obtained, when $h$ is known, through the direct evaluation of the right hand side of equation (d).

Keywords: Interconversion equation, Volterra convolution integral equations, variable Hilbert scales 


\section{INTRODUCTION}

In both the theoretical and practical study of the rheology of linear viscoelastic materials, the interpretation of experimentally measured stress-strain and strain-stress responses are modelled using the linear causal integral equations of viscoelastic relaxation and creep, which are first kind convolution Volterra integral equations the kernels of which are the relaxation and creep moduli $G(t)$ and $J(t)$. Having obtained approximations for $G(t)$ and $J(t)$, they can be used to simulate the stress-strain and strain-stress responses for interesting possibilities without the need to do the actual experimentation. This leads naturally to the need to solve computationally first kind causal Volterra equations. Here, the possibility is examined of using variable Hilbert scales to derive appropriate and representative error estimates.

We will consider computing a function $u \in L_{2}[0, R]$ which is the solution of a first kind convolution Volterra integral equation Volterra (1896)

$$
k * u=\int_{0}^{t} k(t-s) u(s) d s=f(t), \quad 0 \leq t<\infty,
$$

for a given right-hand side $f \in H_{0}^{1}\left(\mathbb{R}_{+}\right)$. It is well-known that, in general, such Volterra integral equations are ill-posed (e.g. Engl et al. (1996)). Furthermore, this integral operator is unbounded as a mapping from $L_{2}(\mathbb{R})$ into itself. Here, we consider the bounded situation by limiting attention to mappings from $L_{2}[0, R]$ into itself, which is, in particular, the situation for the examples considered below.

A frequently used method for solving such Volterra integral equations of the first kind reduces to solving the following second kind Volterra integral equation counterpart of (1)

$$
u(t)+\int_{0}^{t} \frac{k^{\prime}(t-s)}{k(0)} u(s) d s=\frac{1}{k(0)} \frac{d f(t)}{d t},
$$

which is obtained by differentiating (1) with respect to $t$, with the prime / denoting such differentiation. Here, we only consider the case where $k(0) \neq 0$. The ill-posedness is now confined to the determination of the derivative of $f$. However, one also needs to be able to differentiate the kernel $k$. The error associated with this differentiation, if it must be computed numerically, might be considerable. In practice, this method is often used when these derivatives exist and can be computed explicitly (e.g. pages 6 and 20-22 in Brunner and Houwen (1986) and pages 311-323 in Volterra (1896) or pages 8 and 1893-1899 in Volterra (1956)).

A second method, which is the one considered here, is appropriate if one is able to compute a solution $h(t)$ to the interconversion equation (Ferry (1980); Anderssen et al. (2008); de Hoog and Anderssen (2010); Loy and Anderssen (2014))

$$
\int_{0}^{t} k(t-s) h(s) d s=t, \quad 0 \leq t<\infty .
$$

In such situations, the solution takes the following form

$$
u(t)=\frac{d^{2}}{d t^{2}}\left\{\int_{0}^{t} h(t-s) f(s) d s\right\} .
$$

Computing its derivative explicitly gives

$$
u(t)=h(0) \frac{d f(t)}{d t}+h^{\prime}(0) f(t)+\int_{0}^{t} h^{\prime \prime}(t-s) f(s) d s .
$$

Instead of having to perform the numerical differentiation of the kernel $k$ in equation (1) and then solve the resulting second kind Volterra integral equation, or the numerical differentiation of equation (4) after determining $h(t)$, the solution can be obtained, when $h$ is known, through the direct evaluation of the right hand side of equation (5). To obtain $h$ involves solving the interconversion equation which is also ill-posed. However, this does not pose a problem if attention is limited to kernels $k$ for which the interconversion equation can be solved explicitly, which is a situation which occurs in many application areas including rheology.

The method based on the interconversion equation is also convenient for the determination of moments of the solution which can be reformulated using integration by parts as

$$
\int_{0}^{\infty} g(t) u(t) d t=-\int_{0}^{\infty} g^{\prime \prime}(t) \cdot(h * f)(t) d t .
$$


It turns out that if we can compute the second derivative of $g$ exactly, the problem of determining the value of this integral from (observational) the data $f(t)$ is not ill-posed.

Problems where the interconversion equation can be solved explicitly includes

$$
k(t)=1+\sum_{j=1}^{n} a_{j} \exp \left(-t / \tau_{j}\right)
$$

with the $\tau_{j}>0$. It will be shown below, for such $k(t)$, the kernels $h(t)$ which solve the interconversion equation (3) take the following form

$$
h(t)=1+\sum_{j=1}^{n} b_{j} \exp \left(-t / \tau_{j}^{*}\right) .
$$

For future research, we plan to consider the more general kernels of the form

$$
k(t)=1+\int_{0}^{\infty} \exp (-t / \tau) d \mu(\tau)
$$

where $\mu$ corresponds to a positive finite measure.

\section{THE ALGEBRA OF SOME VOLTERRA KERNELS AND THE INTERCONVERSION EQUATION}

Here, we consider the algebra generated by the exponential kernels $k_{\tau}$ defined by

$$
k_{\tau}(t)=\exp (-t / \tau)
$$

where $\tau>0$. Furthermore, let $1=k_{\infty}(t)$ denote the constant kernel. Addition and multiplication with scalars is the usual one for functions of $t$ and the product is the convolution of two kernels $h$ and $k$ defined as

$$
k * h(t)=\int_{0}^{t} k(t-s) h(s) d s=\int_{0}^{t} h(t-s) k(s) d s=h * k(t) .
$$

Note that the product is commutative, but there is no unity. One can show that

$$
k_{\tau} * k_{\sigma}=\frac{\tau \sigma}{\sigma-\tau}\left(k_{\sigma}-k_{\tau}\right)
$$

for the case where $\tau \neq \sigma$ and, in particular, that

$$
k_{\tau} * 1=\tau\left(1-k_{\tau}\right)
$$

Finally, one has

$$
k_{\tau} * k_{\tau}(t)=t k_{\tau}(t)
$$

and, thus, $1 * 1(t)=t$. However, the set of all linear combinations of $k_{\tau}(t)$ is not an algebra with respect to convolution, since the autoconvolution of $k_{\tau}(t)$ is $t k_{\tau}(t)$.

We now consider two kernels $k$ and $h$ defined by

$$
k_{n}(t)=1+\sum_{j=1}^{n} a_{j} k_{\tau_{j}}
$$

and

$$
h_{n}(t)=1+\sum_{i=1}^{n} b_{i} k_{\tau_{i}^{*}}
$$


such that for all $i, j$ one has $\tau_{j} \neq \tau_{i}^{*}$. Then, on using these two relationships, it can be shown that

$$
\begin{aligned}
k_{n} * h_{n}(t)=t+\sum_{j=1}^{n} a_{j} \tau_{j}+\sum_{i=1}^{n} b_{i} \tau_{i}^{*} & +\sum_{i=1}^{n} k_{\tau_{i}^{*}} b_{i} \tau_{i}^{*}\left(\sum_{j=1}^{n} \frac{a_{j} \tau_{j}}{\tau_{i}^{*}-\tau_{j}}-1\right) \\
& +\sum_{j=1}^{n} k_{\tau_{j}} a_{j} \tau_{j}\left(\sum_{i=1}^{n} \frac{b_{i} \tau_{i}^{*}}{\tau_{j}-\tau_{i}^{*}}-1\right) .
\end{aligned}
$$

Since the functions 1 (constant), $t, k_{\tau_{j}}$ and $k_{\tau_{i}^{*}}$ are linearly independent, it follows that

Proposition 1. For the $h_{n}$ and $k_{n}$ defined above, the interconversion equation $h_{n} * k_{n}(t)=t$ holds iff

$$
\begin{aligned}
& \sum_{j=1}^{n} \frac{a_{j} \tau_{j}}{\tau_{i}^{*}-\tau_{j}}=1, \quad i=1, \ldots, n, \quad \text { and } \\
& \sum_{i=1}^{n} \frac{b_{i} \tau_{i}^{*}}{\tau_{j}-\tau_{i}^{*}}=1, \quad j=1, \ldots, n .
\end{aligned}
$$

Proof. One shows that $\sum_{j=1}^{n} a_{j} \tau_{j}+\sum_{i=1}^{n} b_{i} \tau_{i}^{*}=0$ simply by taking $b_{i} \tau_{i} *$ times the first condition, summing over $i$ and adding the sum of $a_{j} \tau_{j}$ times the second condition.

Remark 1. Let $\hat{f}$ denote the Laplace transform of $f$; namely,

$$
\hat{f}(z)=\int_{0}^{\infty} \exp (-z t) f(t) d t
$$

The algebra introduced above is isomorphic to the algebra generated by

$$
\hat{k}_{\tau}(z)=\frac{1}{z-\frac{1}{\tau}}
$$

and one has $\hat{k}_{\infty}(z)=z^{-1}$. Here, the ordinary product corresponds to the convolution in the case of the kernels. This isomorphism is obtained via the Laplace transform. The interconversion equation in this space of rational functions is then

$$
\hat{h}_{\tau}(z) \cdot \hat{k}_{\tau}(z)=\frac{1}{z^{2}}
$$

where $\hat{k}$ is

$$
\hat{k}_{\tau}(z)=\frac{1}{z}+\sum_{j=1}^{n} a_{j} \hat{k}_{\tau_{j}}(z)
$$

and similarly

$$
\hat{h}_{\tau}(z)=\frac{1}{z}+\sum_{i=1}^{n} b_{i} \hat{k}_{\tau_{i}^{*}}(z)
$$

\section{VARIABLE HILBERT SCALES AND ERROR ANALYSIS}

Here, we discuss the error that arises when solving the Volterra integral equation (1) computationally using regularisation. We initially formulate an appropriate functional analytic framework in which to perform the computational error analysis. The Hilbert space of square integrable functions $\mathcal{H}=L_{2}[0, R]$, with $0<R<$ $\infty$, represents such a framework. In addition, let $A \in \mathcal{L}(\mathcal{H})$ be a bounded first kind convolution Volterra integral operator with kernel $k(t)$ such that, for all $u \in \mathcal{H}$, it has the form

$$
A u(t)=\int_{0}^{t} k(t-s) u(s) d s, \quad t \in[0, R] .
$$


Furthermore, let $B$ and $G \in \mathcal{L}(\mathcal{H})$ denote two such Volterra integral operators which satisfy

$$
B A=G
$$

and have kernels $h(t)$ and $g(t)=t$, respectively. Let $L$ denote the following densely defined unbounded linear operator

$$
L u(t)=\frac{d^{2}}{d t^{2}} u(t)
$$

It follows then that $L G=\mathrm{id}$ and consequently the solution of $A u=f$, on the basis of equation (4), is given by

$$
u=L B f \text {. }
$$

The operator $A^{-1}=L B$ is unbounded and one thus requires regularisation to solve the problem numerically despite the fact that one has an explicit formula. In order to be able to deal with relatively general linear regularisation techniques and source conditions, we will utilse the variable Hilbert scales in $\mathcal{H}$ generated by the operator $T=-L$. This is the natural choice in the present situation, yet it is somewhat different to the usual choice $T=\left(A^{*} A\right)^{+}$for compact operators $A$ (Nair et al. (1997); Hegland and Anderssen (2011)).

Recall that the variable Hilbert scales are based on

$$
(u, v)_{\phi}=(u, \phi(T) v)
$$

for some continuous function $\phi(t) \geq C>0$ defined for $t>0$ (an index function). This defines a scalar product on a dense subspace $\mathcal{H}_{\phi} \subset \mathcal{H}$. One then has the variable Hilbert scale interpolation inequality

$$
\|u\|_{\phi \theta} \leq\|u\|_{\theta} \sqrt{\Psi\left(\|u\|_{\psi \theta}^{2} /\|u\|_{\theta}^{2}\right)}
$$

where $\phi, \psi, \theta$ are index functions and $\Psi$ is a convex index function satisfying $\phi(t) \leq \Psi(\psi(t))$.

Using this interpolation inequality, one obtains the following error bound Hegland and Hofmann (2011)

$$
\left\|u_{\alpha}-u\right\| \leq C_{1} \Psi\left(C_{2} / \epsilon\right) \epsilon=\eta(\epsilon) \epsilon,
$$

where $\epsilon$ is a bound for the error in the data and numerical approximations and $u_{\alpha}$ is a regularised solution.

4 NUMERICAL RECONSTRUCTION OF THE SOLUTION $u(t)$ FROM OBSERVED DATA $y_{j}=f\left(t_{j}\right)+\epsilon_{j}$.

In this section, we use equation (5) to numerically compute the solution to $k * u=f$, where we have been given sampled data of $f$ on an equidistant grid which in addition does have some error. We choose the kernel $k$ to be

$$
k(t)=1+a_{1} e^{-t / \tau_{1}}+a_{2} e^{-t / \tau_{2}}
$$

where $a_{1}=0.25, a_{2}=0.75$ and $\tau_{1}=0.2$ and $\tau_{2}=2.5$. The exact solution of (1) in this example has been chosen to be $u(t)=t^{4} e^{-2 t}$. The two scales $\tau_{1,2}$ reflect the behaviour of the kernel around 0 and $\infty$, respectively. We consider a grid $t_{j}=j \Delta t$ with $\Delta t=0.05$ and $j=0, \ldots, 200$. The data is then

$$
y_{j}=k * u\left(t_{j}\right)+\epsilon_{j}
$$

where the $\epsilon_{j}$ are independent and normally distributed with expectation zero and standard deviation $\sigma=0.01$. Using the formula (5), we recover the values of the solution $u\left(t_{j}\right)$ of the integral equation at the points $t_{j}$. As mentioned earlier, we will use the $h$ which is the exact solution of the interconversion equation $k * h(t)=t$. The first term of equation (5) is

$$
h^{\prime}(0) f\left(t_{j}\right) \approx h^{\prime}(0) y_{j} .
$$

The second term is approximated with the trapezoidal rule as

$$
\int_{0}^{t_{j}} h^{\prime \prime}\left(t_{j}-s\right) f(s) d s \approx \frac{\Delta t}{2}\left(h^{\prime \prime}\left(t_{j}\right) y_{0}+2 \sum_{k=1}^{j-1} h^{\prime \prime}\left(t_{j}-t_{k}\right) y_{k}+h^{\prime \prime}(0) y_{j}\right) .
$$




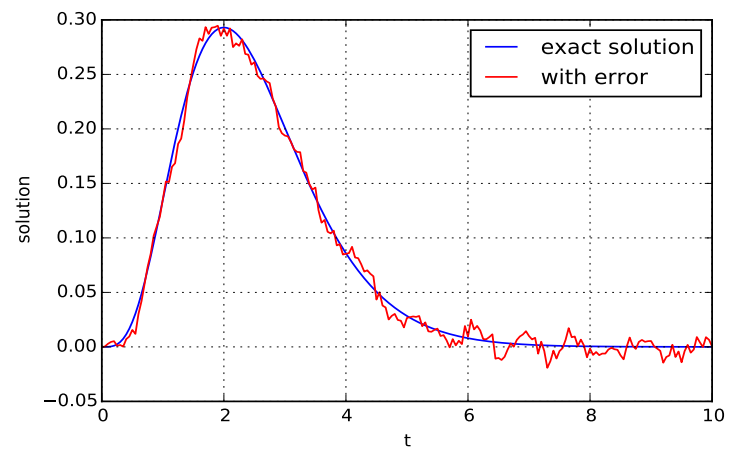

Figure 1. Exact solution and numerical solution from data with error

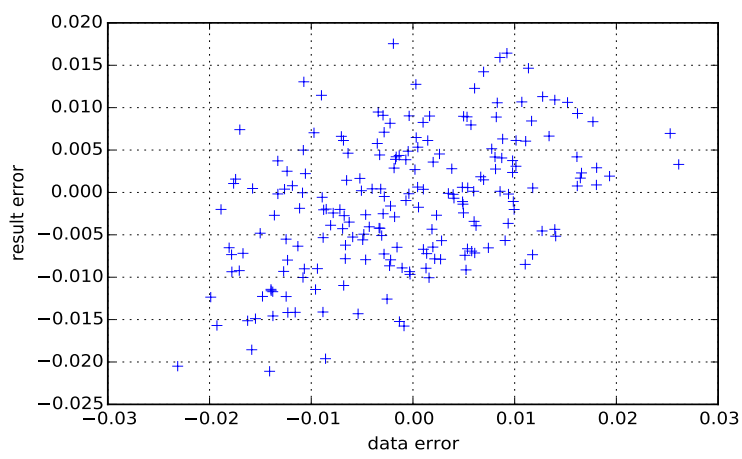

Figure 2. Error of the numerical sollution against data error

The most challenging part is the third term which is approximated as

$$
h(0) \frac{d f}{d t}\left(t_{j}\right) \approx h(0)(D y)_{j}
$$

using the regularised finite difference quotient (Anderssen et al. (1998)).

$$
(D y)_{j}=\frac{1}{q(q+1) \Delta t} \sum_{k=1}^{q}\left(y_{j+k}-y_{j-k}\right)
$$

As this is only defined for $j \geq q$ and $j \leq n-q$, we will set the undefined values to zero. The regularisation parameter $q$ is here chosen to be $q=2$. In practice, however, this parameter needs to be chosen carefully trading-off approximation against data errors using, for example, cross validation. We plan to do a more extensive error analysis in future research. For here, we just consider the variance of the three terms in the formula given that the data does have a variance of $\sigma^{2}$. The more comprehensive error analysis will also include the numerical errors which depend on the smoothness of the exact solution (the source condition).

The standard deviation of the first term of the approximation is then

$$
\sigma_{1}=\sigma\left|h^{\prime}(0)\right|
$$

For the second term, we use the fact that all the errors are i.i.d. and that the trapezoidal rule approximates the integral to give an approximate upper bound for standard deviation for the second term as

$$
\sigma_{2} \leq \sigma \sqrt{\Delta t \int_{0}^{T} h^{\prime \prime}(s)^{2} d s} .
$$


The standard deviation of the third term uses again the fact that the errors of the $y_{j}$ are i.i.d. to give

$$
\sigma_{3}=\sigma \frac{\sqrt{2}|h(0)|}{q^{1 / 2}(q+1) \Delta t} .
$$

Note that the three variances $\sigma_{1,2,3}$ are independent of the discretisation parameter $\Delta t$, proportional to $(\Delta t)^{1 / 2}$ and to $(\Delta t)^{-1 / 2}$, respectively. If the regularisation parameter $q$ is chosen to be proportional to $(\Delta t)^{-2 / 3}$ then the method is stable. For our example and choice of parameters, we obtain to one digit $\sigma_{1}=0.4 \sigma, \sigma_{2}=0.1 \sigma$, and $\sigma_{3}=3 \sigma$.

\section{CONCLUSIONS}

For future research, we plan to consider the more general kernels of the form

$$
k(t)=1+\int_{0}^{\infty} \exp (-t / \tau) d \mu(\tau) .
$$

\section{ACKNOWLEDGEMENT}

While doing this research, the authors have greatly benefitted from discussions with Rick Loy, ANU and Frank de Hoog, CSIRO.

\section{REFERENCES}

Anderssen, B., F. d. Hoog, and M. Hegland (1998). A stable finite difference ansatz for higher order differentiation of non-exact data. Bulletin of the Australian Mathematical Society 58(2), 223-232.

Anderssen, R. S., A. R. Davies, and F. R. de Hoog (2008). On the Volterra integral equation relating creep and relaxation. Inverse Problems 24, 035009 (p. 13).

Brunner, H. and P. J. Houwen (1986). The numerical solution of Volterra equations, Volume 3. Elsevier Science Ltd.

de Hoog, F. R. and R. S. Anderssen (2010). Kernel perturbations for Volterra convolution integral equations. J. Integral Eqn. Appl. 22, 427-441.

Engl, H. W., M. Hanke, and A. Neubauer (1996). Regularization of Inverse Problems. Kluwer Academic Publishers, Dordrecht.

Ferry, J. (1980). Viscoelastic Properties of Polymers. Wiley, New York.

Hegland, M. and R. S. Anderssen (2011). Dilational interpolatory inequalities. Maths Comp. 80, 1019-1036.

Hegland, M. and B. Hofmann (2011). Errors of regularisation under range inclusions using variable Hilbert scales. Inverse Probl. Imaging 5(3), 619-643.

Loy, R. J. and R. S. Anderssen (2014). Interconversion relationships for completely monotone functions. SIAM J. Math. Anal. 46(3), 2008-2032.

Nair, M., M. Hegland, and R. Anderssen (1997). The trade-off between regularity and stability in Tikhonov regularization. Mathematics of Computation of the American Mathematical Society 66, 193-206.

Volterra, V. (1896). Sulla inversione degli integrali definiti, Volume 31. Atti Accad. Sci. Torino.

Volterra, V. (1956). Opere matematiche. Memorie e note. Vol. II. Accademia Nazionale dei Lincei, Roma. 